\title{
A study on the correlation between STAT-1 and mutant p53 expression in glioma
}

\author{
WENBO YANG, HONGWEI WANG, HAITAO JU and CHANGWU DOU
}

\begin{abstract}
Department of Neurosurgery, The Affiliated Hospital of Inner Mongolia Medical University, Hohhot, Inner Mongolia 010055, P.R. China
\end{abstract}

Received January 16, 2016; Accepted January 5, 2017

DOI: $10.3892 / \mathrm{mmr} .2018 .8796$

\begin{abstract}
Glioma is the most common primary brain tumor in adults and the second most common malignant tumor in children. Aberrant expression of signal transducer and activator of transcription 1 (STAT-1) and p53 are known to affect the occurrence and progression of malignant tumors. The aim of the present study was to investigate the expression of STAT-1 and mutant p53 gene, as well as their correlation, in patients with glioma. The present study included 50 patients who underwent glioma resection at the First Affiliated Hospital of Inner Mongolia Medical University between December 2007 and December 2011, and 10 patients with acute cerebral contusion who underwent intracerebral hematoma removal at the same hospital between January 2013 and January 2014. The expression of STAT-1 and mutant p53 protein in patients with different grades of glioma was assessed by immunohistochemistry. Spearman's correlation coefficient was employed to examine the correlation between STAT-1 and the grade of glioma, and mutant p53 expression. The results demonstrated that the mean expression of STAT-1 in glioma was significantly lower compared with normal brain tissue $(\mathrm{P}<0.05)$. However, there was no significant difference in the STAT-1 positive expression rate between the two groups $\left(\chi^{2}=1.38, \mathrm{P}>0.05\right)$. The expression score $(\mathrm{P}<0.05)$ and positive expression rate $\left(\chi^{2}=31.27, \mathrm{P}<0.05\right)$ of mutant p53 in glioma was significantly higher compared with those in normal brain tissue. Statistical analysis revealed a negative correlation between STAT-1 expression and the grade of glioma $(\mathrm{r}=-0.767, \mathrm{P}<0.05)$. In addition, mutant p53 expression was negatively correlated with STAT-1 expression in glioma $(\mathrm{r}=-0.876, \mathrm{P}<0.05)$. The observed negative correlation between STAT-1 and the pathological grade of glioma suggested an association between STAT-1 and the occurrence and development of glioma, thus revealing the
\end{abstract}

Correspondence to: Dr Changwu Dou, Department of Neurosurgery, The Affiliated Hospital of Inner Mongolia Medical University, 5 Xinhua Street, Huimin, Hohhot, Inner Mongolia 010055, P.R. China E-mail: knq7605911@126.com

Key words: STAT-1, p53, glioma, correlation, prognosis potential of STAT-1 as a diagnostic biomarker and therapeutic target for glioma.

\section{Introduction}

Glioma is the most common primary brain tumor in adults and the second most common malignant tumor in children, with an overall annual incidence rate of 3-8/100,000. According to the histological tumor classification standard established by the World Health Organization (WHO), glioma can be classified into four grades (I-IV) and three histological types, namely astrocytoma, oligodendroglioma and oligoastrocytoma (1). Despite the advances in surgical resection, chemotherapy and radiotherapy, the prognosis of patients with glioma remains poor, resulting in an overall 5-year survival rate of $<10 \%$. Currently, glioma ranks third worldwide as the cause of cancer-associated mortality, after pancreatic cancer and lung cancer (2). The standard therapy for newly diagnosed glioma is surgical resection followed by adjuvant radiotherapy and/or chemotherapy. However, surgical resection is often inappropriate due to the unclear boundaries between the primary tumor and adjacent anatomical structures. Furthermore, tumor cells can often rapidly develop resistance to radiotherapy and chemotherapy, leading to low therapeutic efficacy. In addition, therapeutic effects may be affected by the blood-brain barrier and the adverse effects of anticancer drugs. Therefore, the development of novel, effective therapies with fewer adverse effects for the treatment of glioma is imperative.

In recent years, gene therapy using small-molecule inhibitors has garnered attention. It has been reported that members of the signal transducer and activator of transcription (STAT) family are involved in various regulatory processes, such as cellular proliferation, differentiation and apoptosis (3). STAT-1, which was the first member of the STAT family to be discovered, is able to inhibit cell proliferation and promote apoptosis (3). STAT-1 has been identified as a tumor-inhibiting factor, and reduced STAT-1 expression is associated with the occurrence and progression of malignant tumors (4). In addition, it has been demonstrated that the activation of STAT-1 expression in tumor cells by exogenous or endogenous stimuli can improve the sensitivity of these cells to chemotherapy (5). The p53 gene has been classified as a tumor suppressor gene that can effectively inhibit cellular growth and response to stress in various cell types by inducing temporary or 
permanent suppression of proliferation, or by activating cell death processes. It has previously been suggested that the quinoline derivative chloroquine induces apoptosis in human glioma cells by activating the p53 pathway (6), suggesting the potential of p53-based cancer therapies. Furthermore, a p53-dependent activation pathway of STAT1 has been identified (7), indicating a strong association between STAT1 and p53. However, the association between STAT1 and p53 in glioma has not been clarified

Wild-type p53 has been well recognized as a tumor suppressor gene, and p53 is the most frequently mutated gene in human cancer. Evidence has demonstrated that mutant p53 proteins not only lose the ability to suppress tumorigenesis but also gain tumor-promoting functions (8). Therefore, the present study examined the expression of STAT-1 and mutant p53 in glioma of different grades, and analyzed their correlation in order to investigate their effects on the pathogenesis and development of glioma.

\section{Materials and methods}

Subjects. The present study included 50 patients who underwent glioma resection at the First Affiliated Hospital of Inner Mongolia Medical University (Hohhot, China) between December 2007 and December 2011. The patients comprised 29 men and 21 women, with a mean age of 55 years (range, 43-70 years). All glioma specimens were evaluated by an experienced neuropathologist based on the histological classification standard for tumors of the central nervous system established by the WHO in 2007 (1). When the pathological type of a specimen was between grade I-II, grade II-III or grade III-IV, it was classified as belonging to the higher grade. As a result, there were 10 cases of grade I, 14 cases of grade II, 14 cases of grade III and 12 cases of grade IV glioma. A total of 10 patients with acute cerebral contusion, without other nervous system diseases, who underwent intracerebral hematoma removal at the same hospital between January 2013 and January 2014 were selected as controls. The control group comprised 6 men and 4 women with a mean age of 52 years (range, 48-65 years). Brain tissue was collected during the surgery and stored at $-70^{\circ} \mathrm{C}$ until further use.

The study was approved by the Ethics Committee of the First Affiliated Hospital of Inner Mongolia Medical University. Written informed consent was obtained from all patients prior to enrollment in the present study.

Reagents. Rat anti-human STAT-1 polyclonal antibody (cat.no. sc-592) and anti-mutant p53 antibody (cat.no. ab32049) were purchased from Abcam (Cambridge, UK). Antibody dilution buffer was purchased from Wuhan Boster Biological Technology, Ltd. (Wuhan, China). UltraSensitive ${ }^{\mathrm{TM}}$ S-P kits (C reagent, biotinyated sheep anti-mouse immunoglobulin $\mathrm{G}$; $\mathrm{D}$ reagent, streptavidin-peroxidase complex), antigen retrieval solution, hematoxylin and eosin staining solution, and peroxidase/diaminobenzidine (DAB) chromogen (rabbit/mouse) were purchased from Fuzhou Maixin Biotech Co., Ltd. (Fuzhou, China).

Immunohistochemical detection of STAT-1 and mutant p53 expression. All tissue samples were fixed with $10 \%$ formalin for $24 \mathrm{~h}$ at room temperature, embedded in paraffin, and sliced into $4-\mu \mathrm{m}$ sections. The sections were mounted, cleared 3 times with xylene, and rehydrated through serial ethanol dilutions (100, 90, 80, and 70\%) and Tris-buffered saline. Subsequently, the sections were heated in antigen retrieval solution (Fuzhou Maxim Biotech, Inc.) in a pressure cooker for $5 \mathrm{~min}$. The sections were incubated in $3 \%$ hydrogen peroxide for $30 \mathrm{~min}$ to block endogenous peroxidases, and goat serum (Fuzhou Maxim Biotech, Inc.) was used to minimize non-specific binding. The sections were then incubated with rabbit anti-human STAT-1 antibody (1:350 dilution) and anti-mutant p53 antibody (1:300 dilution) at $37^{\circ} \mathrm{C}$ for $1 \mathrm{~h}$. Chromogenic detection of the substrate was performed using the UltraSensitive ${ }^{\mathrm{TM}}$ S-P kit according to the manufacturer's protocol, followed by color development with DAB. The sections were counterstained with hematoxylin, and examined for the extent and intensity of nuclear and non-nuclear staining using a light microscope by two independent observers in a blinded manner.

Determination of positive results. Nuclear and/or cytoplasmic yellow or brown staining was identified as positive staining. Under x200 magnification, 4 observation fields were randomly chosen for each section. A proportion score, which described the estimated percentage of positively-stained tumor cells ( 0 , none; $1,<10 \% ; 2,10-50 \% ; 3,50-80 \% ; 4,>80 \%$ ), and an intensity score, representing the estimated staining intensity $(0$, no staining; 1 , weak; 2 , moderate; 3 , strong), was determined for each sample. A final immunohistochemical score was obtained by multiplying the proportion score and the intensity score, and STAT-1 and mutant p53 expression was classified into 4 subgroups: 0 , negative (-); $1-4$, weakly positive $(+) ; 5-8$, moderately positive $(++)$; and 9-12, strongly positive (+++).

Statistical analysis. All statistical analyses were performed using the SPSS statistical package, version 13.0 (SPSS, Inc., Chicago, IL, USA). Correlations between STAT-1 expression and pathological glioma grades, and between STAT-1 and mutant p53 expression were assessed using Spearman's correlation coefficient. Differences among groups were compared by one-way ANOVA followed by a Tukey's multiple comparison test. The expression ratio of STAT-1 and mutant p53 was compared by $\chi^{2}$ test. $\mathrm{P}<0.05$ was considered to indicate a statistically significant difference.

\section{Results}

Expression characteristics of STAT-1 and mutant p53 in glioma and normal brain tissue. STAT-1 was mainly expressed in the cytoplasm of glioma cells, with some expression also present in the nucleus (Fig. 1). Intense STAT-1 staining was observed in tumor-associated macrophages; however, no staining was present in microglia and capillary endothelial cells. As shown in Table I, although no significant difference was detected between the positive expression rate in normal brain tissue and that in glioma $\left(\chi^{2}=1.38, \mathrm{P}>0.05\right)$, the expression score in glioma $(5.32 \pm 3.25)$ was significantly lower compared with that in normal brain tissue $(10 \pm 1.76, \mathrm{P}<0.05)$. Mutant p53 expression was stronger in the nucleus and was associated with yellow, tan or brown staining (Fig. 2). The positive expression rate and expression score of 
Table I. Expression of STAT-1 in glioma and normal brain tissue.

STAT-1

\begin{tabular}{lccc}
\cline { 2 - 4 } Group & Positive $(\mathrm{n})$ & Positive expression rate $(\%)$ & Expression score $(\mathrm{mean} \pm \mathrm{SD})$ \\
\hline Glioma & 46 & 92 & $5.32 \pm 3.25^{\mathrm{a}}$ \\
Normal brain tissue & 10 & 100 & $10.00 \pm 1.76$ \\
P-value & & $>0.05$ & $<0.05$ \\
$\chi^{2}$ & & 1.38 &
\end{tabular}

${ }^{a} \mathrm{P}<0.05$, compared with normal brain tissue. STAT-1, signal transducer and activator of transcription 1; SD, standard deviation.
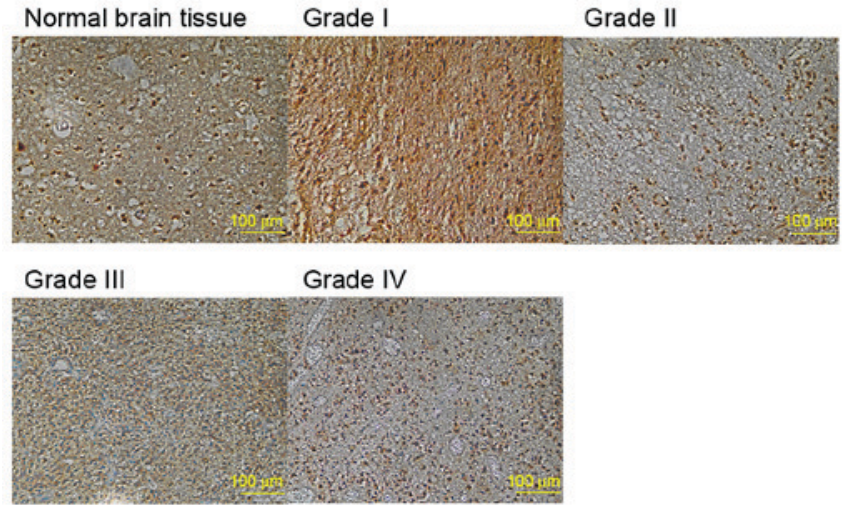

Figure 1. Immunohistochemical staining of STAT-1. STAT-1 expression was detected in normal brain tissue and glioma of different grades using streptavidin-peroxidase staining (magnification, x200). STAT-1 expression was strongly positive in normal brain tissue (+++) and grade I glioma (+++), moderately positive in grade II glioma $(++)$, weakly positive in grade III glioma (+), and negative in grade IV glioma (-). STAT-1, signal transducer and activator of transcription 1.

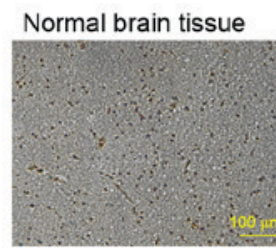

Grade I

Grade II

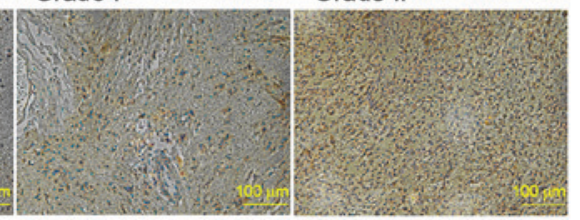

Grade III

Grade IV

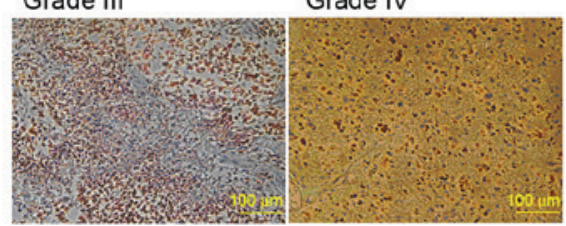

Figure 2. Immunohistochemical staining of mutant p53. Mutant p53 expression was detected in normal brain tissue and glioma of different grades using streptavidin-peroxidase staining (magnification, x200). Mutant p53 expression was negative in normal brain tissue (-), weakly positive in grade I glioma $(+)$, moderately positive in grade II glioma $(++)$, and strongly positive in grade III and IV glioma $(+++)$.

mutant p53 were significantly higher in glioma compared with normal brain tissue $\left(\chi^{2}=31.27, \mathrm{P}<0.05\right)$, as shown in Table II.

Correlation between STAT-1 expression and glioma grade. As shown in Table III, the expression score of STAT-1 in glioma was reduced as the tumor grade increased. Spearman's correlation analysis (Table IV) revealed a negative correlation between STAT-1 expression and tumor grade $(\mathrm{r}=-0.767$, $\mathrm{P}<0.01)$.

Correlation between STAT-1 and mutant p53 expression in glioma. The expression score of mutant p53 in glioma is presented in Table III. The mutant p53 expression score in glioma (grade I-IV) was significantly higher compared with the control group $(\mathrm{P}<0.05)$. Furthermore, the mutant p53 expression score in grade II, III and IV glioma was significantly higher than in grade I glioma $(\mathrm{P}<0.01)$. Spearman's correlation analysis (Table $\mathrm{V}$ ) revealed a negative correlation between STAT-1 and mutant p53 expression in glioma $(r=-0.876$, $\mathrm{P}<0.01)$.

\section{Discussion}

The gene coding for STAT-1 has been classified as a tumor suppressor gene. A previous study demonstrated that STAT-1 overexpression in squamous cell carcinoma inhibits cell growth (9), whereas transfection with STAT-1 was revealed to inhibit the proliferation and promote the apoptosis of U87MG cells in vitro (10). These results suggested that STAT-1 may participate in inducing the apoptosis of glioma cells. Furthermore, STAT-1 inhibits the activity of proliferating cell nuclear antigen by stimulating p21 expression, thus suppressing DNA replication and cell cycle progression in U87MG cells, and decreasing in vitro cell survival. In addition, a mouse study demonstrated that STAT-1 defects inhibit interferon (IFN)-mediated immune responses to bacteria and viruses. Conversely, transfection with STAT-1 may result in a significant decline in the growth and metastatic rate of highly tumorigenic cancer cells in nude mice (11).

Most of the antitumor effects of radiotherapy and chemotherapy are mediated by the cell cycle checkpoint, a mechanism normally activated to repair DNA damage. Apoptosis of damaged cells is activated when DNA damage cannot be repaired. In a previous study regarding ataxia telangiectasia mutated (ATM) checkpoint pathways, STAT-1 was reported to upregulate the transcription of two important intermediary factors, namely p53 connexin 1 (53BP1) and DNA damage checkpoint 1 (MDC1) (12). In the present study, STAT-1 expression was reduced in patients with a higher glioma grade, whereas it has previously been demonstrated that the effects of 
Table II. Expression of mutant p53 in glioma and normal brain tissue.

\begin{tabular}{lccc}
\hline & \multicolumn{2}{c}{ Mutant $\mathrm{p} 53$} \\
\cline { 2 - 4 } Group & Positive (n) & Positive expression rate (\%) & Expression score (mean \pm SD) \\
\hline Glioma & 46 & 92 & $3.10 \pm 1.28^{\mathrm{a}}$ \\
Normal brain tissue & 6 & 60 & $0.80 \pm 0.79$ \\
P-value & & $<0.05$ & $<0.05$ \\
$\chi^{2}$ & & 31.27 & \\
\hline
\end{tabular}

${ }^{\mathrm{a}} \mathrm{P}<0.05$, compared with normal brain tissue. $\mathrm{SD}$, standard deviation.

Table III. Expression scores of STAT-1 and mutant p53 in glioma of different grades.

\begin{tabular}{lccc}
\hline Group & Case number & STAT-1 expression scores (mean \pm SD) & Mutant p53 expression scores (mean \pm SD) \\
\hline Control & 10 & $10.00 \pm 1.76$ & $0.80 \pm 0.79$ \\
Glioma & & & $1.20 \pm 0.79^{\mathrm{a}}$ \\
Grade I & 10 & $8.50 \pm 2.07^{\mathrm{a}}$ & $3.14 \pm 2.44^{\mathrm{c}, \mathrm{d}}$ \\
Grade II & 14 & $6.93 \pm 3.08^{\mathrm{a}, \mathrm{b}}$ & $3.93 \pm 2.56^{\mathrm{c}, \mathrm{d}}$ \\
Grade III & 14 & $3.21 \pm 2.08^{\mathrm{c}-\mathrm{e}}$ & $3.67 \pm 1.87^{\mathrm{c}, \mathrm{d}}$ \\
Grade IV & 12 & $3.17 \pm 1.99^{\mathrm{c}-\mathrm{e}}$ &
\end{tabular}

STAT-1, signal transducer and activator of transcription 1 ; SD, standard deviation. ${ }^{\mathrm{a}} \mathrm{P}<0.05$ compared with control group, ${ }^{\mathrm{b}} \mathrm{P}<0.05$ compared with grade I glioma group, ${ }^{\mathrm{C}}<0.01$ compared with control group, ${ }^{\mathrm{d}} \mathrm{P}<0.01$ compared with grade I glioma group, ${ }^{\mathrm{e}} \mathrm{P}<0.05$ compared with grade II group.

Table IV. Correlation analysis of STAT-1 expression and different grades of glioma.

\begin{tabular}{lccc}
\hline Comparison & $\mathrm{n}$ & $\begin{array}{c}\text { Correlation } \\
\text { coefficient }\end{array}$ & $\begin{array}{l}\text { Bilateral } \\
\text { P-value }\end{array}$ \\
\hline Grade vs. STAT-1 & 60 & $-0.767^{\mathrm{a}}$ & $<0.01$ \\
\hline $\begin{array}{l}\text { Spearman's correlation coefficient was used to examine the correlation. } \\
\text { a'Correlation is significant when } \mathrm{P}<0.05 .\end{array}$ & \\
\hline
\end{tabular}

Table V. Correlation analysis of STAT-1 and mutant p53 expression.

\begin{tabular}{lccc}
\hline Comparison & $\mathrm{n}$ & $\begin{array}{c}\text { Correlation } \\
\text { coefficient }\end{array}$ & $\begin{array}{c}\text { Bilateral } \\
\text { P-value }\end{array}$ \\
\hline STAT-1 vs. mutant p53 & 60 & $-0.876^{\mathrm{a}}$ & $<0.01$ \\
\hline
\end{tabular}

Spearman's correlation coefficient was used to examine the correlation. ${ }^{\text {a}}$ Correlation is significant when $\mathrm{P}<0.05$.

ATM on tumor radiosensitivity are decreased with increasing glioma grade. Therefore, it may be hypothesized that decreased STAT-1 expression results in reduced tumor radiosensitivity via the ATM checkpoint. Similar studies have revealed that STAT-1-positive cancer may improve chemosensitivity of the tumor $(10,13,14)$, whereas STAT-1 activation by IFN- $\alpha / \beta$ and antineoplastic drugs may exert a synergistic effect on the induction of apoptosis (5). In addition, the functions of the topoisomerase I inhibitor irinotecan on squamous cell carcinoma, and those of the antimetabolite raltitrexed, appear to depend on the expression of STAT-1 (15), thus suggesting that similar mechanisms may exist in glioma.

STAT-1 and anticancer drugs may interact through cell cycle checkpoints, a possibility that needs to be addressed in further studies. Numerous anticancer drugs trigger the DNA damage checkpoint and exert their antineoplastic effects through the induction of DNA damage and genotoxic stress, as well as through the activation of ATM serine/threonine kinase and ataxia telangiectasia and Rad3-related protein. A previous study suggested that STAT-1 participates in ATM checkpoint activation through upregulating two important mediators, namely 53BP1 and MDC1, a finding that proposes a role for STAT-1 in enhancing the effect of DNA-damaging agents through activation of ATM checkpoint pathways (16).

STAT-1 can also be used as an indicator for the efficacy of immunotherapy, since STAT-1 defects are associated with immune escape mechanisms exploited by invasive tumors (17). Since some antineoplastic drugs are able to enhance the antitumor immune response (18), the higher chemosensitivity of STAT-1-expressing gliomas may be associated with the activation of immune surveillance mechanisms by STAT-1 signaling pathways (19). In addition, a high percentage of patients with low intratumoral STAT-1 expression exhibited reduced sensitivity to adjuvant chemotherapy. In the present study, 
the negative correlation between STAT-1 expression and the pathological grade of glioma suggested that STAT-1, as well as being a valuable biomarker for evaluating the degree of glioma malignancy, also exhibits potential as a molecular target for enhancing the radio- and chemosensitivity of tumors.

The p53 gene is a tumor suppressor gene located on chromosome 17 in humans. Although the pathogenesis of glioma remains to be elucidated, studies have suggested that it is associated with p53 gene mutation or loss $(20,21)$. It has also been demonstrated that although p53 mutations may be present regardless of the glioma grade, the mutation rate in high-grade glioma is significantly higher compared with that in low-grade glioma (22). The p53 gene can temporarily or permanently suppress cell growth, or activate stress-associated cell death mechanisms, thus providing a theoretical basis for p53-based cancer therapy (23). In addition, a role for p53 in the radioand chemosensitivity of tumors has been suggested $(24,25)$. A recent study regarding gene therapy targeting p53 revealed that survival rates of the human glioma cell line U87MG were markedly reduced following transfection with the wild-type p53 gene using an adenoviral vector (26). In addition, another study reported that gliomas of a higher grade exhibited increased mutant p53 gene expression (27), thus suggesting an association between mutant p53 gene expression and the development and progression of glioma. p53 holds potential as a target for gene therapies that aim to increase the sensitivity of glioma cells to chemotherapy or radiotherapy.

Anticancer drugs, such as fludarabine, doxorubicin and cisplatin, are known to activate the tumor suppressor gene p53 (28). Furthermore, it has been confirmed that STAT-1 and p53 synergistically promote tumor cell death (29). Notably, anticancer drugs can activate STAT-1 in p53-positive cells, but fail to work in p53-null cells (7), thus suggesting that p53 serves a necessary role in STAT-1 activation. This finding is further supported by studies demonstrating that anti-p53 small interfering RNAs, as well as overexpression of the mouse double minute 2 homolog (MDM2) gene, coding the E3 ubiquitin-protein ligase $\mathrm{Mdm} 2$, which is a negative regulator of p53, can deactivate STAT-1 expression (29). Overall, these findings suggested that STAT-1 activation by genotoxic drugs depends on p53 (7). The present study revealed a negative correlation between STAT-1 and mutant p53 expression in glioma cells, indicating a crucial role of both proteins in the occurrence and development of glioma.

In conclusion, the present study revealed a negative correlation between the expression of the STAT-1 gene and the glioma grade, as well as between STAT-1 and mutant p53 expression. The negative correlation between STAT-1 and the pathological level of glioma suggested that STAT-1 may be associated with the occurrence and development of glioma, and may be a diagnostic biomarker and therapeutic target for the malignancy of glioma.

\section{Acknowledgements}

The authors of the present study would like to thank all the members of the Inner Mongolia Medical Molecular Pathology Laboratory for their contribution to this experiment. This study was funded by the Inner Mongolia Natural Science Foundation Project (grant nos. 2014MS0836 and 2014MS08121).

\section{Competing interests}

The authors declare that they have no competing interests.

\section{References}

1. Louis DN, Ohgaki H, Wiestler OD, Cavenee WK, Burger PC, Jouvet A, Scheithauer BW and Kleihues P: The 2007 WHO classification of tumors of the central nervous system. Acta Neuropathol 114: 97-109, 2007

2. Xu L, Li Z, Tao Y, Li RH, Fang F, Zhao H, Li G, Li YH, Wang J, Feng X and Pan J: Histone acetyltransferase inhibitor II induces apoptosis in glioma cell lines via the p53 signaling pathway. J Exp Clin Cancer Res 33: 108, 2014.

3. Kim HS and Lee MS: STAT1 as a key modulator of cell death. Cell Signal 19: 454-465, 2007.

4. Bruyère C, Madonna S, Van Goietsenoven G, Mathieu V, Dessolin J, Kraus JL, Lefranc F and Kiss R: JLK1486, a Bis 8-hydroxyquinoline-substituted benzylamine, displays cytostatic effects in experimental gliomas through MyT1 and STAT1 activation and, to a lesser extent, PPAR $\gamma$ activation. Transl Oncol 4: 126-137, 2011

5. Strobl B and Moriggl R: Recovery from chemotherapy depends on STAT1 for replenishment of B lymphopoiesis. J Leukoc Biol 95: 849-851, 2014.

6. Kim EL, Wüstenberg R, Rübsam A, Schmitz-Salue C, Warnecke G, Bücker EM, Pettkus N, Speidel D, Rohde V, Schulz-Schaeffer W, et al: Chloroquine activates the $\mathrm{p} 53$ pathway and induces apoptosis in human glioma cells. Neuro Oncol 12: 389-400, 2010.

7. Youlyouz-Marfak I, Gachard N, Le Clorennec C, Najjar I, Baran-Marszak F, Reminieras L, May E, Bornkamm GW, Fagard R and Feuillard J: Identification of a novel p53-dependent activation pathway of STAT1 by antitumour genotoxic agents. Cell Death Differ 15: 376-385, 2008.

8. Yue X, Zhao Y, Xu Y, Zheng M, Feng Z and Hu W: Mutant p53 in cancer: Accumulation, gain-of-function, and therapy. J Mol Biol 429: 1595-1606, 2017.

9. Xi S, Dyer KF, Kimak M, Zhang Q, Gooding WE, Chaillet JR, Chai RL, Ferrell RE, Zamboni B, Hunt J and Grandis JR: Decreased STAT1 expression by promoter methylation in squamous cell carcinogenesis. J Natl Cancer Inst 98: 181-189, 2006.

10. Ju H, Li X, Li H, Wang X, Wang H, Li Y, Dou C and Zhao G: Mediation of multiple pathways regulating cell proliferation, migration, laboratory investigation. J Neurosurg 118: 1239-1247, 2013.

11. Huang S, Bucana CD, Van Arsdall $M$ and Fidler IJ: Stat1 negatively regulates angiogenesis, tumorigenicity and metastasis of tumor cells. Oncogene 21: 2504-2512, 2002.

12. Barry SP, Townsend PA, Knight RA, Scarabelli TM, Latchman DS and Stephanou A: STAT3 modulates the DNA damage response pathway. Int J Exp Pathol 91: 506-514, 2010.

13. Thomas M, Finnegan CE, Rogers KM, Purcell JW, Trimble A, Johnston PG and Boland MP: STAT1: A modulator of chemotherapy-induced apoptosis. Cancer Res 64: 8357-8364, 2004.

14. Zhu H, Wang Z, Xu Q, Zhang Y, Zhai Y, Bai J, Liu M, Hui Z and $\mathrm{Xu} \mathrm{N}$ : Inhibition of STAT1 sensitizes renal cell carcinomacells to radiotherapy and chemotherapy. Cancer Biol Ther 13: 401-407, 2012.

15. McDermott U, Longley DB, Galligan L, Allen W, Wilson T and Johnston PG: Effect of p53 status and STAT1 on chemotherapy-induced, Fas-mediated apoptosis in colorectal cancer. Cancer Res 65: 8951-8960 2005.

16. Duan X,Ponomareva L, Veeranki S, Panchanathan R, Dickerson E and Choubey D: Differential roles for the interferon-inducible IFI16 and AIM2 innate immune sensors for cytosolic DNA in cellular senescence of human fibroblasts. Mol Cancer Res 9: 589-602, 2011.

17. Liu K, Caldwell SA and Abrams SI: Immune selection and emergence of aggressive tumor variants as negative consequences of Fas-mediatedcytotoxicity and altered IFN-gamma-regulated gene expression. Cancer Res 65: 4376-4388, 2005.

18. Lake RA and Robinson BW: Immunotherapy and chemotherapy-a practical partnership. Nat Rev Cancer 5: 397-405, 2005.

19. Dunn GP, Koebel CM and Schreiber RD: Interferons, immunity and cancer immunoediting. Nat Rev Immunol 6: 836-848, 2006

20. Watanabe T, Katayama Y, Yoshino A, Komine C and Yokoyama T: Deregulation of the TP53/p14ARF tumor suppressor pathway in low-grade diffuse astrocytomas and its influenceon clinical course. Clin Cancer Res 9: 4884-4890, 2003. 
21. Wang YY, Zhang T, Li SW, Qian TY, Fan X, Peng XX, Ma J, Wang $\mathrm{L}$ and Jiang T: Mapping p53 mutations in low-grade glioma: A voxel-based neuroimaging analysis. AJNR Am J Neuroradiol 36: 70-76, 2015.

22. Cohen AL and Colman H: Glioma biology and molecular markers. Cancer Treat Res 163: 15-30, 2015.

23. Bell HS and Ryan KM: Targeting the p53 family for cancer therapy: 'Big brother' joins the fight. Cell Cycle 6: 1995-2000, 2007.

24. Fedrigo CA, Grivicich I, Schunemann DP, Chemale IM, dos Santos D, Jacovas T, Boschetti PS, Jotz GP, Braga Filho A and da Rocha AB: Radioresistance of human glioma spheroids and expression of HSP70, p53 and EGFr. Radiat Oncol 6: 156, 2011.

25. Biddlestone-Thorpe L, Sajjad M, Rosenberg E, Beckta JM, Valerie NC, Tokarz M, Adams BR, Wagner AF, Khalil A, Gilfor D, et al: ATM kinase inhibition preferentially sensitizes p53 mutant glioma to ionizing radiation. Clin Cancer Res 19: 3189-3200, 2013.
26. Lang FF, Yung WK, Raju U, Libunao F, Terry NH and Tofilon PJ: Enhancement of radiosensitivity of wild-type p53 human glioma cells by adenovirus-mediated delivery of the p53 gene. J Neurosurg 89: 125-132, 1988.

27. Broaddus WC, Liu Y, Steele LL, Gillies GT, Lin PS, Loudon WG, Valerie K, Schmidt-Ullrich RK and Fillmore HL: Enhanced radiosensitivity of malignant glioma cell after adenoviral p53 transduction. J Neurosurg 91: 997-1004, 1999.

28. Xiang W, Zhu XL and Zhao HY: Therapeutic effect of p53 gene combined with cisplatin on experimental glioma. Chin J Clin Neurosur 11: 154-156, 2006 (In Chinese).

29. Townsend PA, Scarabelli TM, Davidson SM, Knight RA, Latchman DS and Stephanou A: STAT-1 interacts with p53 to enhance DNA damage-induced apoptosis J Biol Chem 279: 5811-5820, 2004. 